\title{
Successful use of albuterol in a patient with central core disease and mitochondrial dysfunction
}

\author{
L. T. W. Schreuder • M. W. G. Nijhuis-van der Sanden • \\ A. de Hair • G. Peters • S. Wortmann • L. A. Bok • \\ E. Morava
}

Received: 25 November 2009 /Revised: 14 March 2010 / Accepted: 22 March 2010 /Published online: 5 May 2010

(C) The Author(s) 2010. This article is published with open access at Springerlink.com

\begin{abstract}
Albuterol, a selective beta-adrenergic agonist, has been used experimentally in combination with exercise therapy in a few inherited neuromuscular disorders to increase muscle strength and muscle volume. We report on a 9-year-old boy with central core disease and mitochondrial dysfunction due to compound heterozygous RYR1 mutations receiving albuterol treatment for 1 year. Throughout the period of albuterol administration, the patient underwent an aerobic exercise regime of training sessions three times a week that lasted $20 \mathrm{~min}$ each. No side effects of albuterol use were seen. Significant clinical progress, including self care, sitting up, raising arms above the shoulders, independent feeding, and better speech and writing were observed compared with minimal development of these abilities in the previous years
\end{abstract}

Communicated by: Shamima Rahman

References to electronic databases

Central core disease: OMIM\#117000

Fascioscapulohumoral muscular dystrophy: OMIM\#158900

Spinal muscular atrophy: OMIM\#253300/253400/253550/271150

RYR1: ryanodine receptor 1 (skeletal), geneID 6261

POLG: polymerase (DNA-directed) gamma, geneID 5428

Competing interest: None declared.

L. T. W. Schreuder $\cdot$ S. Wortmann $\cdot$ E. Morava $(\bowtie)$

Departments of Pediatrics and Neurology,

Nijmegen Centre for Mitochondrial Disorders,

Radboud University Nijmegen Medical Centre,

P.O Box 9101, 6500 HB Nijmegen, The Netherlands

e-mail: E.Morava@cukz.umcn.nl

M. W. G. Nijhuis-van der Sanden · G. Peters

Department of Paediatric Physical Therapy,

Radboud University Nijmegen Medical Centre,

Nijmegen, The Netherlands

A. de Hair · L. A. Bok

Pediatric Department, Máxima Medical Center Veldhoven,

Veldhoven, The Netherlands on physiotherapy. Improved forced expiratory volume in $1 \mathrm{~s}$ $\left(\mathrm{FEV}_{1}\right)$ score was detected and increased muscle strength was noted: progress was measured using various functional tests and assessment scales. The only complication observed was a mild progression of the joint contractures, possibly due to an unbalance between the flexor and extensor musculature. In general, in this pilot study in a complex case of metabolic myopathy our patient has shown promising results following albuterol treatment and aerobic exercise therapy.

\section{Abbreviations \\ CMAS Childhood Myositis Assessment Scale \\ FSHD Fascioscapulohumeral muscular dystrophy}

\section{Introduction}

Inactivity in patients with muscle disease in combination with a primary or secondary mitochondrial dysfunction might cause a further decrease in muscle mass and muscle tone and a further decrease in adenosine triphosphate (ATP) production (Finsterer 2004). This deteriorating cascade of restriction in mobility leads to diminished function and decrease in quality of life (QOL) by increasing isolation (Morava et al. 2006). Patients with exercise intolerance and muscle weakness could benefit from regular exercise. Benefits could include improvement of activity, general fitness, and some increase in muscle strength. However, there is little evidence for the benefit of exercise in congenital myopathies. In mitochondrial disorders, strength training could be even deleterious due to increased lactate production and muscle damage. On the other hand, aerobic training in these patients appears to be beneficial. The results in patients carrying highly heteroplasmic mitochondrial DNA mutations are still controversial; some studies 
report a relative increase of mutation frequency in a second biopsy after a fitness therapy period of 6 months to 1 year (Janssen et al. 2006, Phoenix et al. 2006).

Recently, albuterol has been used experimentally in a few inherited neuromuscular disorders in order to increase muscle strength and muscle volume. Albuterol is a selective betaadrenergic agonist used to control asthma. Albuterol is contraindicated in case of hypertension, heart disease, hyperthyroidism, and diabetes mellitus, restricting the use of the drug in endocrine and metabolic myopathies. In facioscapulohumeral muscular dystrophy (FSHD; OMIM 158900), the combined use of albuterol and exercise training had a positive effect on muscle strength and volume, with an increase in the lean body mass (Kissel et al. 1998, 2001). In patients diagnosed with spinal muscular atrophy (SMA; OMIM 253300/253400/253550/271150), the use of albuterol leads to a significantly higher myometry, improvement in the forced vital capacity (FVC), and increase in lean body mass without adverse effects (Hirano 2003; Kinali et al 2002).

Multi/minicore myopathy (OMIM 117000) is a congenital myopathy presenting with generalized muscle hypotonia, decreased muscle strength, decreased deep tendon reflexes, and a stagnatory muscle disease. Many patients eventually show a very slow clinical improvement, but in some cases, difficulties increase over time (Jungbluth 2007). Most patients have contractures in the knees, hips, and shoulders, hindering eventual exercise, and children with the disorder are prone to frequent respiratory infections and hypoventilation. Apart from the ryanodine 1 (RYRI; gene ID 6261) gene, another gene is implicated in the disease. If the selenoprotein $\mathrm{N}$ (SEPN; gene ID 57190) gene is impaired, patients are more likely to develop pulmonary problems. This is not a prominent feature in RYR1-related multi/minicore myopathy (Jungbluth 2007): due to mutations to the ryanodine receptor 1 (RYR1; gene ID 6261) gene, leakage of the ryanodine receptor may occur, causing chronic elevation of cytosolic calcium (Gommans et al. 2002). In the cells' periphery, plasma membrane calcium-channel $\left(\mathrm{Ca}^{2+}\right)$ pumps and sodium $\left(\mathrm{Na}^{+}\right) / \mathrm{Ca}^{2}$ exchangers remove this calcium, whereas mitochondria and sarcoplasmic reticulum $\mathrm{Ca}^{2+}$ adenosine triphosphate (ATP)ase (SERCA) are responsible for nearly all removal from the center of the cell (Gommans et al. 2002). Due to absence of pumps and exchangers in the center of the cell, there is elevated calcium, which causes most of the damage to mitochondria. Therefore, patients with multi/minicore myopathy might present with mitochondrial dysfunction (Wortmann et al. 2009), in turn causing lower ATP synthesis, which can lead to disorganization of the myofibrils, leading to pathologic central cores and muscle weakness (Loke and MacLennan 1998). Albuterol increases the level of the fast-twitch isoform of SERCA (SERCA1a) (Zhang et al. 1996). Changes in the levels of SERCA proteins, particularly SERCA1a, could account for increasing calcium uptake rate observed in homogenates of muscles from the albuterol-treated animals (Zhang et al. 1996). This would increase the ability to clear intracellular calcium, resulting in improved muscle function and less damage to mitochondria.

In previous studies, the experimental use of albuterol in this patient group resulted in increased Medical Research Council (MRC) scores, myometry, and FVC in patients receiving a 6-month trial period with regular physiotherapy (Messina et al. 2004). However, no data on the metabolic parameters of these patients were reported. In adults with a metabolic myopathy due to mitochondrial DNA mutations, aerobic exercise training has been used with positive outcome (Cejudo et al. 2005; Jeppesen et al. 2006; Joseph et al. 2006; Taivassalo et al. 2006). Here we describe the clinical course of a patient diagnosed with central-core myopathy and mitochondrial dysfunction on aerobic exercise and albuterol treatment. This is the same patient as the one reported in 2009 by Wortmann and colleagues (Wortmann et al. 2009).

\section{Patient and methods}

\section{Treatment protocol}

After approval of the ethical commission and obtaining informed consent, albuterol was administered to our patient four times a day for 1 year at a dosage of $2 \mathrm{mg}$ each time. To monitor the occurrence of possible cardiac side effects, cardiological evaluation consisting of electrocardiogram (ECG) and cardiac echography took place every 3 months $\left(\mathrm{T}_{0}, \mathrm{~T}_{1}, \mathrm{~T}_{2}, \mathrm{~T}_{3}\right.$, and $\mathrm{T}_{4}$ ). In addition, weekly measurements of heart rate, blood pressure, and blood glucose values were performed and the patient's weight monitored. In addition to administration of the drug, the patient underwent 20-min aerobic training exercise three times a week. This training was aimed at the quadriceps muscles, flexors and extensor of the hip and lower arm, biceps brachii muscles, and musculature of the shoulder's rotator cuff. The maximum number of repeats for each exercise was established by the physiotherapist. Apart from these protocol-based sessions, functional activities were performed daily (practicing getting dressed, throwing, catching, "soccer", sitting upright, rolling over in bed, and putting shoes on). To evaluate progress, the patient underwent physiotherapeutic evaluation at $\mathrm{T}_{0}, \mathrm{~T}_{2}$, and $\mathrm{T}_{4}$. During these evaluations, he was evaluated clinically. The motometrical instruments used to document his progress included the functional scale according to Scott, modified by Bäckman, which is a measurement tool for muscle function (Bäckman 1988; Scott et al. 1982); the forced expiratory volume in $1 \mathrm{~s}\left(\mathrm{FEV}_{1}\right)$ as a measurement of respiratory function associated with increasing strength of respiratory muscles, the Childhood Myositis Assessment Scale (CMAS) 
for quantitative evaluation of muscle function (Huber et al. 2004), and the Jamar dynamometer for assessment of force for various muscle groups (Trutschnigg et al. 2008). Concerning the functional scale according to Scott et al. (1982) and the CMAS, it should be taken into account that some items could not be scored because of the patient's inability to stand up, which is a prerequisite for these items. Therefore, the patient could never be expected to reach the maximum score of 52. The scales were merely documented to quantify the patient's increasing functionality.

\section{Patient}

The patient was the second child of nonconsanguineous parents who was born after an uneventful pregnancy of 42 weeks. At birth, early findings comprised congenital hypotonia, low facial expression, and inverted feet. He was diagnosed with swallowing problems and gastroesophageal reflux. Dysmorphic features included brachyturricephaly, low-set ears, hypermetropia, facies myopathica, and hyperlaxity with arachnodactyly. Due to positive family history of minicore myopathy (his sister died with this condition at age 5 due to aspiration), an early muscle biopsy was performed. The initial histological findings suggested a possible multi/minicore disease. The patient developed chronic lactic acidemia (lactate $2.3-4 \mathrm{mmol} / \mathrm{l}, \mathrm{C}$ : $<2.1 \mathrm{mmol} / \mathrm{l})$, 3-methylglutaconic aciduria (80 $\mu \mathrm{mol} / \mathrm{l}, \mathrm{C}$ : $<18 \mu \mathrm{mol} / \mathrm{mmol}$ creatinine), and recurrent hypoglycemic episodes. Serum alanine levels $(610 \mu \mathrm{mol} / \mathrm{l}, \mathrm{C}:<450 \mu \mathrm{mol} / \mathrm{l})$ and creatine kinase (CK) levels (500-800 U/1, C: $180 \mathrm{U} / \mathrm{l})$ were moderately increased. No motor development was observed with regular physiotherapy; for further clarification of the diagnosis, a second muscle biopsy was performed at the age of 6 years. The biopsy results showed the characteristic picture of central core disease: electron microscopy detected abnormal, large mitochondria with crystalline inclusions and biochemical evidence of severe mitochondrial dysfunction (Table 1). Genetic studies including mitochondrial DNA sequencing, sequencing of the nuclear-coded structural complexes I and III genes, and POLG [polymerase (DNA directed) gamma; gene
ID 5428] mutation analysis were all normal. A compound heterozygous RYR1 mutation in complementary DNA (cDNA) [Val48491le+1739-1741 dup, with premature stop in position 610 (1741insATCAfsX29)] was found. The parents were heterozygous carriers (Wortmann et al. 2009). At age 9 years, while receiving regular physiotherapy, the boy could not sit up, raise his arms above the level of the hips, hold a pen, or stand due to his severe, generalized muscle weakness and contractures. Further physical signs were bilateral ptosis, facies myopathica with open mouth, and kyphoscoliosis in addition to joint contractures, presenting foremost in his hips and knees. He had no extraocular muscle anomalies and no exercise-induced myalgia.

\section{Results}

At the age of 9 years $\left(\mathrm{T}_{0}\right)$, the patient was unable to come to a sitting position on his own and required assistance. He could not raise his arms above his head and was unable to keep his arms stretched before him. After the start of the trial, improvements in functionality, independence, and spontaneous movements were reported even after 6 weeks without any complications or adverse events. At the time of the physiotherapeutic evaluation at $T_{2}$, (after 6 months of therapy), there was a striking increase in strength, and the patient followed the aforementioned training regime with compliance and great enthusiasm. After 1 year of treatment, at $\mathrm{T}_{4}$, significant further improvement became evident, including several functional aspects in fine-motor development (turning a key, holding a pen, brushing teeth), activity (active movements throughout the day), and speech development (decreasing dysarthria; less pronounced facies myopathica and ptosis). At this point, he could sit up straight without assistance, turn over in bed, and come to a sitting position on his own from lying down. Also, he could raise his arms above his head and even execute careful punches on a boxing ball on a pole. Results for the functional scale according to Scott et al. (1982), FEV ${ }_{1}$ and
Table 1 Abnormal biochemical and genetic features

ATP adenosine triphosphate, $U C$ unconditioned stimulus, $N$ normal

\begin{tabular}{ll}
\hline Analyses performed & Results \\
\hline Biochemistry & \\
ATP production nmol/h/mUCS (N 42-81) & 4 \\
Complex I mU/UCS (N 70-251) & 58 \\
Complex II mU/UCS (N 335-749) & 312 \\
Complex III mU/UCS (N 2200-6610) & 725 \\
Complex IV mU/UCS (N 810-3120) & 483 \\
Complex deficiencies & I, II, III, IV \\
Genetics & \\
Paternal mutation & p. His581GlnfsX29 (exon 16) \\
Maternal mutation & p.Val14849Ile (exon 101) \\
\hline
\end{tabular}


Table 2 Motometric scores

\begin{tabular}{llll}
\hline & Scott et al. 1982) & FEV $_{1}$ & CMAS \\
\hline $\mathrm{T}_{0}$ & 6 & 1,46 & 3 \\
$\mathrm{~T}_{2}$ & 9 & 1,81 & 12 \\
$\mathrm{~T}_{4}$ & 9 & 1,85 & 20 \\
\hline
\end{tabular}

$F E V_{l}$ forced expiratory value in $1 \mathrm{~s}, C M A S$ Childhood Myositis Assessment Scale

CMAS are summarized in Table 2, showing increased values for $\mathrm{FEV}_{1}$ and explosive strength. Scores for the dynamometric tests, which illustrate a variable increase in muscle force for different muscle groups, are displayed in Table 3. Values for hip extensor muscles and ankle flexor and extensor muscles could not be provided because of the lack of sensitivity of the measurement instruments at lowforce levels. In the latter two muscle groups, the boy's inverted feet made dynamometric testing impossible.

The only complication observed was that after 1 year of treatment, the boy's flexion contractures increased $15^{\circ}$ in his hips and $30^{\circ}$ in his knees, possibly correlating with the poor knee extensor scores in Table 3. No abnormalities were reported on the evaluations of the patient's cardiac function, heart rate, blood pressure, and blood glucose values. No peculiar changes in body weight were noticed, apart from the normal increase attributed to the patient growing.

\section{Discussion}

In summary, in this pilot study, a complex case of metabolic myopathy (multi/minicore disease and mitochondrial dysfunction), our patient showed promising results on betaadrenergic-agonist treatment and exercise therapy. We report the evident increase in muscle strength and independent functionality as well as improved $\mathrm{FEV}_{1}$ after treatment with albuterol combined with regular aerobic exercise. No serious side effects were seen from the use of the beta agonist. An important point in this accomplishment was the motivation based on early therapeutic success in our patient. Unfortunately, after an initial decrease of joint contractures in the patient's lower extremities, a gradual increase of contractures was reported after 1 year of training, hindering self-care and mobility. As an underlying explanation, we hypothesized the lack of balance between flexor and extensor muscle force, which could cause a passive decline in extensor muscle force.

Aside from his multi/minicore myopathy, our patient had chronic lactic acidemia, a shortened fasting tolerance, 3methyl glutaconic acidemia, and severe mitochondrial dysfunction, with deficiencies in the oxidative phosphorylation complexes I, II, III, and IV. The metabolic abnormalities in patients with central-core myopathy have been suggested to be present due to the underlying cellular transport defect. Central-core disease occurs due to autosomal recessive mutations in the RYRl gene and presents with abnormal mitochondrial structure, detected by histology and electron microscopy (central core due to abnormal mitochondrial structure and localization), due to abnormal cellular calcium transport. Normal calcium transport is essential for normal mitochondrial function (Monnier et al. 2008).

Studies on aerobic exercise therapy have been reported with promising results in several adult patients with mitochondrial dysfunction (Cejudo et al. 2005; Joseph et al. 2006; Messina et al. 2004; Taivassalo et al. 2006). Based on the success in the complex patient reported here, one should therefore hypothesize that anabolic therapy combined with aerobic exercise might useful in treating patients with certain types of mitochondrial myopathies. So far, to
Table 3 Hand-held dynamometer scores

Dynamometric scores for each body half are recorded for each of the physiotherapeutic evaluations. Two measurements were done at each point. Changes in average values compared with the first measured value $\left(T_{0}\right.$, but $T_{2}$ for elbow flexors) are outlined in the columns titled "Increase"

$N D$ no data

\begin{tabular}{|c|c|c|c|c|c|c|c|c|}
\hline \multirow{2}{*}{$\begin{array}{l}\text { Body part } \\
\text { Left }\end{array}$} & \multicolumn{2}{|l|}{$\mathrm{T}_{0}$} & \multicolumn{2}{|l|}{$\mathrm{T}_{2}$} & \multicolumn{3}{|c|}{$\mathrm{T}_{4}$} & \multirow[b]{2}{*}{ Increase } \\
\hline & & & & & Increase & & & \\
\hline Shoulder abduction. & 23 & 21 & 35 & 26 & $+39 \%$ & 31 & 26 & $+30 \%$ \\
\hline Elbow flexion & ND & ND & 17 & 17 & ND & 22 & 17 & $+15 \%$ \\
\hline Elbow extension & 12 & 11 & 16 & 17 & $+43 \%$ & 31 & 31 & $+170 \%$ \\
\hline Hip flexion & 31 & 22 & 47 & 53 & $+89 \%$ & 62 & 62 & $+134 \%$ \\
\hline Knee flexion & 37 & 37 & 42 & 34 & $+24 \%$ & 55 & 44 & $+34 \%$ \\
\hline Knee extension & 17 & 17 & 26 & 37 & $+85 \%$ & 22 & 35 & $+68 \%$ \\
\hline \multicolumn{9}{|l|}{ Right } \\
\hline Shoulder abduction & 17 & 14 & 31 & 22 & $+71 \%$ & 31 & 23 & $+74 \%$ \\
\hline Elbow flexion & ND & ND & 17 & 18 & ND & 17 & 22 & $+11 \%$ \\
\hline Elbow extension & 12 & 11 & 17 & 17 & $+48 \%$ & 22 & 17 & $+70 \%$ \\
\hline Hip flexion & 17 & 17 & 84 & 55 & $+309 \%$ & 93 & 80 & $+409 \%$ \\
\hline Knee flexion & 46 & 42 & 45 & 51 & $+9 \%$ & 53 & 62 & $+31 \%$ \\
\hline Knee extension & 26 & 22 & 31 & 33 & $+33 \%$ & 17 & 17 & $+29 \%$ \\
\hline
\end{tabular}


our knowledge, no prospective study has been performed in children or adults using beta analogs in patients with a primary mitochondrial defect and myopathy. The beneficial effect of beta agonists in treating core myopathy patients has been reported before (Messina et al. 2004). Although mitochondrial function has not been reported in those patients, considering a similar histopathological phenotype, it seems unlikely that the beneficial effect of albuterol in this cohort had a different basis than the beneficial effect observed in this paper.

Open Access This article is distributed under the terms of the Creative Commons Attribution Noncommercial License which permits any noncommercial use, distribution, and reproduction in any medium, provided the original author(s) and source are credited.

\section{References}

Bäckman E (1988) Methods for measurement of muscle function. Methodological aspects, reference values for children, and clinical applications. Scand J Rehabil Med Suppl 20:9-95, Review

Cejudo P, Bautista J, Montemayor T et al (2005) Exercise training in mitochondrial myopathy: a randomized controlled trial. Muscle Nerve 32:342-350

Finsterer J (2004) Mitochondriopathies. Eur J Neurol 11:163-186, Review

Gommans IM, Vlak MH, de Haan A, van Engelen BG (2002) Calcium regulation and muscle disease. J Muscle Res Cell Motil 23(1):59-63, Review

Hirano M (2003) Pilot trial of albuterol in spinal muscular atrophy. Curr Neurol Neurosci Rep 3:56

Huber AM, Feldman BM, Rennebohm RM et al (2004) Validation and clinical significance of the Childhood Myositis Assessment Scale for assessment of muscle function in the juvenile idiopathic inflammatory myopathies. Arthritis Rheum 50:1595-1603

Janssen AJ, Trijbels FJ, Sengers RC et al (2006) Measurement of the energy-generating capacity of human muscle mitochondria: diagnostic procedure and application to human pathology. Clin Chem 52:860-871

Jeppesen TD, Schwartz M, Olsen DB et al (2006) Aerobic training is safe and improves exercise capacity in patients with mitochondrial myopathy. Brain 129:3402-3412
Joseph AM, Pilegaard H, Litvintsev A et al (2006) Control of gene expression and mitochondrial biogenesis in the muscular adaptation to endurance exercise. Essays Biochem 42:13-29, Review

Jungbluth H (2007) Multi-minicore Disease Orphanet J Rare Dis 2:31, Review

Kinali M, Mercuri E, Main M et al (2002) Pilot trial of albuterol in spinal muscular atrophy. Neurology 59:609-610

Kissel JT, McDermott MP, Mendell JR et al (2001) Randomized, double-blind, placebo-controlled trial of albuterol in facioscapulohumeral dystrophy. Neurology 57:1434-1440

Kissel JT, McDermott MP, Natarajan R et al (1998) Pilot trial of albuterol in facioscapulohumeral muscular dystrophy. FSH-DY Group. Neurology 50:1402-1406

Loke J, MacLennan DH (1998) Malignant hyperthermia and central core disease: disorders of $\mathrm{Ca} 2+$ release channels. Am J Med 104(5):470-486, Review

Messina S, Hartley L, Main M et al (2004) Pilot trial of salbutamol in central core and multi-minicore diseases. Neuropediatrics 35:262-266

Monnier N, Marty I, Faure J et al (2008) Null mutations causing depletion of the type 1 ryanodine receptor (RYR1) are commonly associated with recessive structural congenital myopathies with cores. Hum Mutat 29(5):670-678

Morava E, van den Heuvel L, Hol F et al (2006) Mitochondrial disease criteria: diagnostic applications in children. Neurology 67:1823-1826

Phoenix C, Schaefer AM, Elson JL et al (2006) A scale to monitor progression and treatment of mitochondrial disease in children. Neuromuscul Disord 16:814-820

Scott OM, Hyde SA, Goddard C et al (1982) Quantitation of muscle function in children: A prospective study in Duchenne muscular dystrophy. Muscle Nerve 5:291-301

Taivassalo T, Gardner JL, Taylor RW et al (2006) Endurance training and detraining in mitochondrial myopathies due to single largescale mtDNA deletions. Brain 129:3391-3401

Trutschnigg B, Kilgour RD, Reinglas J et al (2008) Precision and reliability of strength (Jamar vs. Biodex handgrip) and body composition (dual-energy X-ray absorptiometry vs. bioimpedance analysis) measurements in advanced cancer patients. Appl Physiol Nutr Metab 33:1232-1239

Wortmann SB, Rodenburg RJ, Jonckheere A et al (2009) Biochemical and genetic analysis of 3-methylglutaconic aciduria type IV: a diagnostic strategy. Brain 132:136-146

Zhang KM, Hu P, Wang SW, Feher JJ, Wright LD, Wechsler AS, Spratt JA, Briggs FN (1996) Salbutamol changes the molecular and mechanical properties of canine skeletal muscle. J Physiol 496(Pt 1):211-220 